\title{
Proton pump inhibitors overuse: only inappropriate prescriptions or further iatrogenic damage?
}

\author{
Mario Visconti \\ Department of Internal Medicine, Santa Maria del Popolo degli Incurabili Hospital, Naples, Italy
}

\begin{abstract}
Proton pump inhibitors (PPIs) are the most potent drugs for reducing gastric acid secretion; so, since their release in the late 1980 s, they have been recommended as the first therapeutic choice for many gastroesophageal diseases, risk reduction in or healing of non-steroidal anti-inflammatory drugs-associated ulcer disease and stress ulcer prophylaxis in intensive care unit patients. Thus PPIs account for a significant proportion of pharmaceutical health-care expenditure. Much of this high expenditure results from overuse of PPIs in account of inappropriate indications or prolongation of therapies for excessive time compared to real need. PPIs overutilization occurs in all medical care settings: in the majority of hospitalized patients with low risks for gastrointestinal bleeding, in patients healed at discharge from hospital, in outpatients in ambulatory practice. However potential adverse effects associated with PPIs therapy have been described, including enteric (especially by Clostridium difficile in elderly patients) and pneumonia infections, nutritional deficiencies, rebound acid hypersecretion, acute interstitial nephritis, gastric neoplasms, bone fractures. Caution is required for some coprescription, particularly with clopidogrel.
\end{abstract}

\section{Introduction}

Proton pump inhibitors (PPIs) are the most potent drugs for reducing gastric acid secretion; thus, since their release in the late 1980s, they have been recommended as the first therapeutic choice for: i) gastroesophageal reflux disease (GERD); ii) non-erosive reflux disease; iii) healing of erosive esophagitis; iv) maintenance of healing of erosive esophagitis; v) peptic ulcer disease; vi) maintenance of healed ulcer disease; vii) Zollinger Ellison syndrome; viii) treatment of Helicobacter pylori infections in combination with antibiotics; ix) risk reduction in and healing of nonsteroidal anti-inflammatory drugs (NSAID)-associated ulcer disease; $x$ ) stress ulcer prophylaxis (SUP)

Correspondence: Mario Visconti, Department of Internal Medicine, Santa Maria del Popolo degli Incurabili Hospital, Rampe S. Giovanni Maggiore 12, 80134 Naples, Italy.

Fax: +39.081 .551539$

E-mail: mario.visconti1@tin.it

Key words: Proton pump inhibitors overuse; proton pump inhibitors unjustified cost; proton pump inhibitors adverse effects.

Received for publication: 21 November 2014.

Accepted for publication: 21 November 2014.

This work is licensed under a Creative Commons Attribution NonCommercial 3.0 License (CC BY-NC 3.0).

(C) Copyright M. Visconti, 2015

Licensee PAGEPress, Italy

Italian Journal of Medicine 2015; 9:217-228

doi:10.4081/itjm.2015.562 in intensive care unit patients; xi) pyrosis and some dyspeptic syndromes as NSAID induced dyspepsia. ${ }^{1}$

Proven the PPIs effectiveness in so widespread pathologic conditions, in recent years there has been a progressive increase in the number of prescriptions. In Germany the PPIs use is risen from 44 million defined daily doses (DDD) in 1993 to 1674 million DDD $(+3804 \%)$ in 2008 with an associated cost of $€ 540$ million. ${ }^{2}$

In USA the data obtained with a 12-month (April 2013 - March 2014) survey on the top 100 drugs by total prescriptions and total sales showed that only levothyroxine and rosuvastatin had a higher number of prescriptions than esomeprazole (respectively, more than 23 million, about 22.9 million and 19.3 million). In March 2014 the esomeprazole cost was US\$ 6.2 billion. ${ }^{3}$ Thus, PPIs account for a significant proportion of pharmaceutical health-care expenditure, even if the decrease in price of these drugs in the last years has partially balanced the substantial increase in drug usage.

The National Institute for Health and Clinical Excellence (NICE) published its guidelines on PPIs in 2000. The recommendations for using these drugs, particularly in the long term, are relatively selective. ${ }^{4}$ Expenditure on PPIs would be far less than $90 \%$ of the total dyspepsia drug budget if restricted to the recommended indications; however, there are many and strong evidences that the well-established guidelines are not often followed. The reasons for this are unclear and cannot be explained solely by increased morbidity, new indications and new diagnostic procedures. Certainly there is also a PPIs overprescription, often explained by the anxiety for possible medico-legal consequences. PPIs overuse has been identified in primary and secondary care and at the 
interface between primary and secondary care, especially at discharge from the hospital. In all different care settings there is also a potential cause for an increasing number of clinical risks. Thus, the economic and clinical consequences of the frequent PPIs inappropriate prescription have often been studied and described in the biomedical literature. In this review a summary of the most important damages due to the use of these drugs without a correct indication or for a not justified long term is expounded, proving that their overutilization is a serious economic and clinical mistake.

\section{Overutilization of proton pump inhibitors therapy in hospitalized medical patients}

In 2013 a research, very interesting for the originality of the methodology, was performed by the Department of Medicine of Waterford Regional Hospital (Ireland). ${ }^{5}$ The aim was to evaluate the appropriateness of PPIs prescription by conducting an audit against recommended guidelines (NICE 2000).

The study participants were 205 consecutive patients admitted to medical wards and assessed for the use of PPIs (mean age 61 years; range 19-95 years). All six medical wards were audited and the prescription charts were surveyed to identify patients on PPIs; this was carried out over a period of two weeks. The study was based on a specific questionnaire. In the first step, patient medical records, such as general practitioner (GPs) letters and hospital visit reports, were reviewed for further data: age, gender, demographic details, dosage and duration of PPIs use, whether PPIs were started during this admission, if PPIs therapy was begun by hospital doctors or GPs, any endoscopies performed to support the diagnosis and any documentation of indication for prescribing PPIs. In the second step, a short interview was carried out with each patient to evaluate patients' knowledge of their treatment by asking the clinical reason for using PPIs; moreover, the interview was carried out to identify possible discrepancies between patients' reports and medical records data, particularly about the procedure and the duration of the therapy. At the end, statistical analysis was performed.

This method of investigation has allowed to obtain a big amount of data; the more interesting are reported in this schematic list: i) $79 \%(162 / 205)$ of patients were using PPIs at admission to the hospital; ii) in $64 \%$ of patients PPIs were prescribed by hospital doctors, either during their current or previous visits; in $36 \%$ of the cases the therapy was instituted by GPs; iii) the duration of PPIs therapy ranged from 1 day to 15 years; $31 \%$ $(51 / 162)$ of patients were taking PPIs for $\geq 2$ years and another $18 \%(\mathrm{n}=29)$ were using PPIs between $1-2$ years; iv) no documentation for PPIs valid indication was found for $45 \%$ (73/162) of patients; v) only $39 \%(n=63)$ of patients were aware of the reason for being prescribed a PPI, and among $27 \%(17 / 63)$ of this group discrepancies were noted between patient report and medical record data; vi) healing or preventing NSAIDs/aspirininduced ulcer was the sole reason for PPIs co-prescription in $20 \%$ (33/162) of the studied patients; vii) to only $14 \%$ (23/162) of patients PPIs recommended maintenance doses were prescribed and significantly the vast majority of patients were using PPIs long-term high doses; viii) only $12 \%(20 / 162)$ of patients had their upper gastrointestinal endoscopies for confirmation or justification for being on PPIs.

Similar results have been described in previous studies and in different countries (USA, Sweden, UK) showing that a PPIs considerably excessive usage does exist in inpatient settings, ${ }^{6,7}$ that the frequency of PPIs discontinuation is low in long-term users, ${ }^{8}$ that there are more difficulties in discontinuing PPIs in GERD than in nonGERD patients ${ }^{8}$ and that an educational intervention employed to reduce PPIs inappropriate prescription in the community had no effect on the proportion of patients taking these drugs at the time of hospital admission. ${ }^{9}$

In addition, the list of Haroon et al. provides another important and exact result: of note, to the majority of medical inpatients PPIs were prescribed with an inappropriate or a poor indication, being lower than expected the number of patients who had endoscopy before starting PPIs therapy.

The guidelines recommend long-term therapy only for few indications, such as gastro-esophageal reflux disease, and for patients with a documented NSAIDsinduced ulcer who cannot avoid a continuous therapy with NSAIDs. However, in this study about $60 \%$ of patients were using long-term high doses of PPIs although the frequent possibility of the interruption of the therapy or the use of lower maintenance doses. A reason for this mistake is certainly the lack of communication between the clinical staff and the patient, who often do not well know the importance and the characteristics of his disease. Thus, a regular monitoring and the re-evaluation by the attending physicians, either in primary or secondary care, of the continuous need for PPIs remains crucial. Moreover, it is very important to educate the patient about his disease, taking into consideration all the changes in the clinical course.

\section{Proton pump inhibitors overutilization for stress ulcer prophylaxis}

Evidence-based guidelines for SUP were published by the American Society of Health-System Pharmacists (ASHP) in $1999 .{ }^{10}$ SUP is not recommended for adult medical and surgical patients admitted in non-Intensive Care Units (ICU) with fewer than two risk factors for clinically significant bleeding. More important factors are sepsis, occult or overt bleeding for 6 or more days, history of gastric ulceration or bleeding during the 12 
months preceding the admission, hepatic failure, spinal cord injury, partial hepatectomy, thermal injury involving greater than $35 \%$ of the body, or head injury with Glasgow coma score of less than or equal to $10 .{ }^{11} \mathrm{SUP}$ is not warranted in patients at low risk for clinically insignificant bleeding (e.g., patients not receiving mechanical ventilation or those without significant coagulopathy). The number-needed-to-treat to prevent a single episode of clinically significant gastrointestinal bleeding in the ICU setting is greater than $900,{ }^{12}$ this data being unknown for the non-ICU setting but expected to be much higher.

Despite a lack of evidence supporting its use, several studies have shown that in the non-ICU setting an antisecretory therapy (AST) for SUP has been prescribed without proper indication to $22-54 \%$ of patients. In most cases AST was with PPIs. ${ }^{13-15}$

A cross-sectional web-based survey in a university-affiliated tertiary care hospital in Massachusetts found that $69 \%$ of physicians prescribed SUP to over $25 \%$ of patients in the non-ICU setting inappropriately fearing an improbable upper gastrointestinal (GI) bleeding [odds ratio (OR) 2.7; 95\% confidence interval (CI) 1.07-7.28] or medico-legal repercussions for not prescribing SUP (OR 3.02; 95\% CI 1.07-8.56). ${ }^{16}$

\section{Proton pump inhibitors overutilization after hospital discharge}

Some careful studies have cumulatively found that over $50 \%$ of inpatients, who started PPIs therapy in the non-ICU setting, was discharged with an unnecessary prescription of PPIs. ${ }^{13-15}$

One retrospective cohort study conducted in a single US academic hospital found that the inpatient costs for SUP in the non-ICU setting were US\$ 44,000 annually. If these costs are coupled with nearly US\$ 68,000 in outpatient pharmacy costs, when the PPIs were reflexively continued upon hospital discharge, combined estimated expenditure was of nearly US\$ 112,000 annually, which could easily be prevented through the proper adherence to SUP guidelines. ${ }^{13}$

Sometimes after hospital discharge the patients continue unnecessary therapy with PPIs without doctor's prescription because they worry to lose the previous treatment advantages. Often PPIs are used for long term and uninterruptedly therapy with a significant increase in costs and risks of adverse events.

\section{Proton pump inhibitors overutilization in ambulatory practice}

Regarding this problematic, better information is obtained from some retrospective or observational studies more than from few adequate trials.
A retrospective medical record review conducted in Ann Arbor to determine the prevalence and economic effect of inappropriate PPIs use in an ambulatory care setting found that of 946 patients categorized according to appropriateness of pharmacotherapy based on documented upper GI tract diagnoses, GI or extraesophageal symptoms, or gastroprotection - $35.4 \%$ were given PPIs therapy for an appropriately documented upper GI tract diagnosis, $10.1 \%$ received PPIs empirically for symptomatic treatment based on extraesophageal symptoms, $18.4 \%$ received PPIs for gastroprotection, and $36.1 \%$ had no documented appropriate indication for PPIs therapy. In a subgroup analysis, $48.6 \%$ of patients across all 4 categories received PPIs without documentation of reevaluation of upper GI tract symptoms, disavowing the potential for on-demand or step-down therapy, and accounting for 1034 patient-years of PPIs use. The total cost of inappropriate PPIs use was US\$ $1,566,252$ based upon average wholesale price costs. ${ }^{17}$

A study conducted through a Boston health plan evaluated, via pharmacy billing data from two insures, prescription patterns of both PPIs and histamine 2 receptor antagonists (H2RA) in patient on chronic therapy ( $>90$ days) in account of AST. In a total study population of 168,727 adult patients a relevant upper GI diagnosis was found only in $61 \%$ of the cases, mainly for dyspepsia ( $42 \%$ of total) and GERD (38\% of total). Thus, approximately $39 \%$ of patients lacked appropriate documentation for any upper GI diagnosis. There was no subgroup analysis with regard to defined gastroprotection with PPIs. Diagnostic testing was uncommon, with only $19 \%$ having undergone esophagogastroduodenoscopy within the prior 2 years. Despite long-term therapy more than $30 \%$ of responders $(59 \%)$ experienced heartburn or reflux more than twice a week and more than half experienced symptoms of dyspepsia at least once a week. A significant proportion of patients on chronic therapy with PPIs or H2RA lacked definitive upper GI diagnoses in their billing data. Thus, PPIs overuse in this outpatient setting was often initiated and continued with undocumented or unsubstantiated diagnoses. The high symptom burden and low use of diagnostic testing indicate opportunities for improvement in the care of patients on chronic AST. ${ }^{18}$

\section{Overutilization of proton pump inhibitors in gastroesophageal reflux disease therapy: some more problems}

As discussed above, GERD is a correct and frequent indication for PPIs therapy (Figure 1).

GERD often needs high dosages to obtain the remission of GI and/or extra-GI symptoms and longterm utilization to keep up clinical comfort. It has 
earlier been shown that a high number of patients, maintained on greater than single-daily dose of PPIs to treat GERD symptoms, could be reduced to singledose therapy without relapse of symptoms. In a prospective cohort study from a single center, $117 \mathrm{pa}$ tients asymptomatic for GERD symptoms were stepped-down from a multiple-dose of PPIs to a single-dose therapy with lifestyle modification. The patients were submitted to an accurate follow up with scheduled controls and possibility to contact the research center at any time. Six months after being stepped-down to a PPIs single-dose, $79.5 \%$ of patients remained free of GERD symptoms. ${ }^{19}$ Of note, 16/19 (84\%) of patients, who failed the step-down during a previous therapeutic attempt, remained free of GERD symptoms after stepping-down during this trial. It is not easy to explain the reasons for this success. It is reasonable that a greater attention to the timing of PPIs dosing (one-half hour before a meal) and a diligent adherence to recommended lifestyle modifications (e.g., smaller portion meals, avoidance of eating for $4 \mathrm{~h}$ before sleep, head of bed elevation) have contributed to the step-down success despite the earlier failure. ${ }^{13}$

Other therapeutic options, which can be usefully alternative to PPIs uninterrupted and long-term use, have been reported:

- On-demand therapy, defined as administration of medication in response to symptoms with discontinuation of drugs after symptoms are alleviated, is an attractive therapeutic option because of costeffectiveness and patient compliance.
Three randomized, placebo controlled trials examined the results of on-demand therapy using different PPIs and different dosages in non-erosive reflux disease patients. The primary end point was the proportion of subjects willing to continue ondemand therapy after 6 months of intervention, which was significatively greater among patients randomized to receive PPIs therapy (83-85\%) versus placebo $(48-56 \%)\left(\mathrm{P}<0.01\right.$ in all studies). ${ }^{20-22}$ Another trial placebo-controlled study showed that $432 / 523(83 \%)$ of patients reported a complete symptom relief with PPIs therapy at the end of the acute phase. During the following on-demand treatment, discontinuation rates because of inadequate heartburn control were $20 \%$ for placebo $v s$ $6 \%$ for PPIs $(\mathrm{P}<0.00001) .{ }^{23}$ Antacid use was twofold higher in the placebo group.

Despite these results, it is possible that sometimes the patients do not comply with the rigorous instructions about the on demand therapy and do not modify the frequency and interval of medication intake, even when the symptoms become significantly less intense. ${ }^{24}$

- Step-off PPIs to less expensive drugs for gastric acid ipersecretion (histamine-2-receptor antagonists) or no GERD drugs (prokinetics) in patients in whom PPIs alleviated GERD symptoms. The independent factor associated with successful stepoff from PPI was age: for every decade of increased age there was a $35 \%$ increase in the likelihood of success. ${ }^{25}$

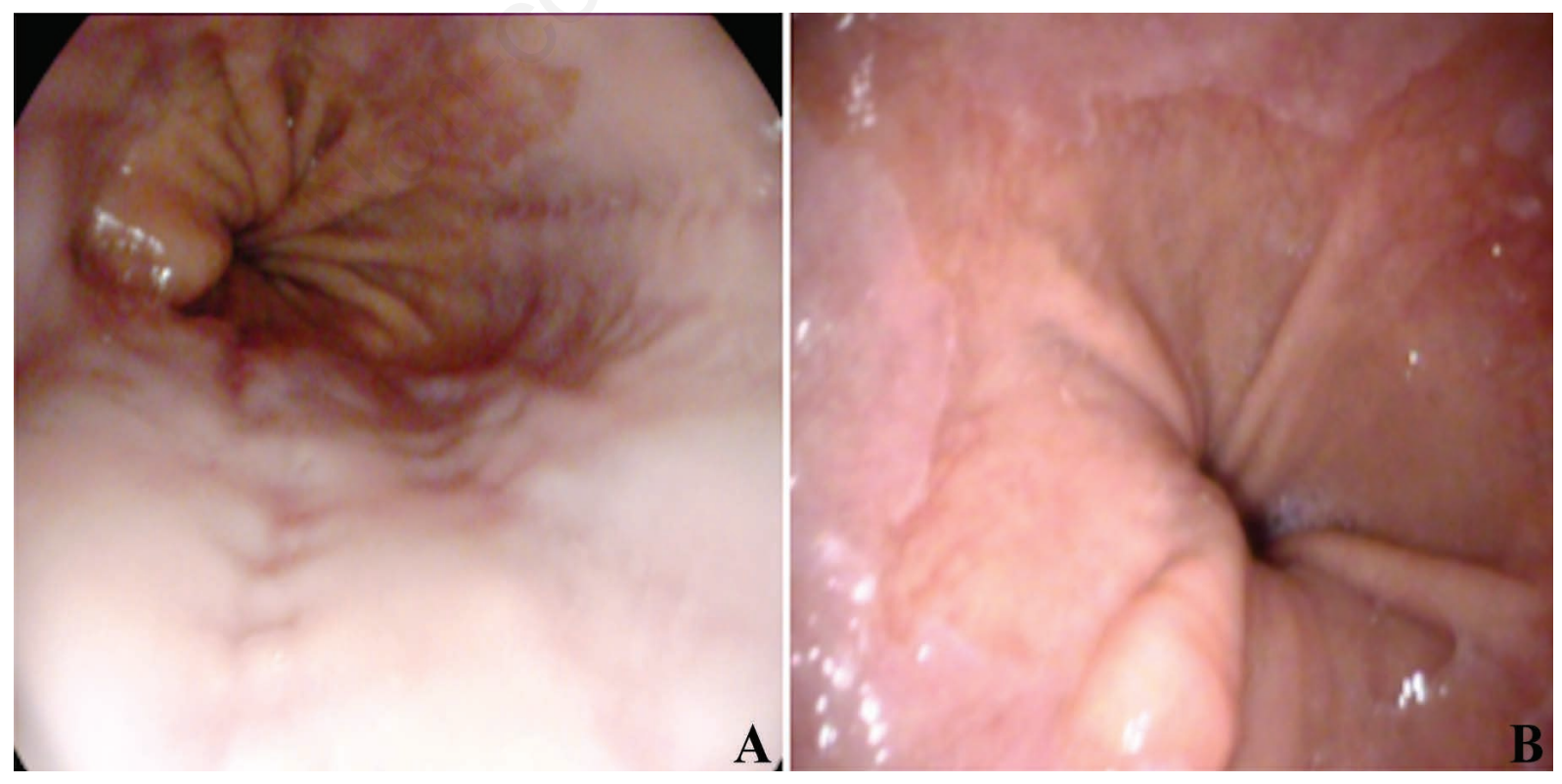

Figure 1. A) Erosive esophagitis before proton pump inhibitors therapy. B) Suspected endoscopic esophageal metaplasia (Barrett's esophagus?). Courtesy by Pietro Di Giorgio and Francesco Giannattasio, Unit of Digestive Endoscopy, Loreto Mare Hospital, Naples, Italy. 
These recommendations about possible alternatives to long-term uninterrupted PPIs therapy have been accepted by a recent revision of NICE guidelines, which replaces NICE clinical guidelines 17 (published August 2004), offering new evidence-based advice on the care and treatment of adults with symptoms of dyspepsia or symptoms suggestive of GERD or both. ${ }^{26}$

\section{Iatrogenic damages by proton pump inhibitors}

Some potential adverse effects associated with PPIs therapy have been described over the past decades, including enteric and pneumonia infections, nutritional deficiencies (B12, iron and magnesium), rebound acid hypersecretion, acute interstitial nephritis, gastric neoplasms, bone fractures. ${ }^{27}$ The majority of the available data are derived from retrospective studies that cannot fully adjust for comorbid conditions or prove a direct cause/effect relationship. In general, patients who receive PPIs therapy (especially hospitalized patients) have more comorbid conditions than those who do not receive therapy, which is a major confounder with respect to outcomes risks discussed below. ${ }^{28}$ All the same it seems obvious that PPIs overutilization, particularly inappropriate long term utilization, can increase incidence and clinical relevance of these effects, sometimes causing more damages than benefits.

\section{Gastrointestinal infections}

The risk of enteric infections associated with PPIs use centers on the theory of impaired destruction of ingested microorganisms by gastric acid as $\mathrm{pH}$ rises above $4 .{ }^{29}$ So achlorydria or severe hypochlorydria, due to chronic atrophic gastritis and long term PPIs administration, may cause an increased risk of enteric infection. A detailed meta-analysis provided evidence linking various durations of PPIs therapy in hospitalized patients with an increased risk of enteric infections by Clostridium difficile (CD), Campylobacter, Salmonella, Shigella, and Listeria. A 2007 meta-analysis was the first to suggest a potential three-fold increased risk for enteric infections, including Salmonella, Campylobacter and Shigella, among PPIs users. ${ }^{30}$

Moreover, although it is thought that PPIs have a minor effect on altering the intestinal bacterial microbiota and observational studies have suggested that PPIs may or may not increase risk of enteric infections, these drugs can cause bacterial overgrowth in the small bowel and consequently the diarrheal disease defined as small intestinal bacterial overgrowth syndrome, sometimes mistakenly diagnosed as irritable bowel syndrome. ${ }^{31,32}$ PPIs not only increase intestinal bacterial colonization but also accelerate intestinal transit.
Greater attention must to be given to CD infection, which may cause the pseudomembranous colitis, a disease with various levels of severity, sometimes mortal.

In 2012, physicians were alerted to a two- to threefold increased risk of $C$. difficile acute disease (CDAD) among elderly patients with chronic comorbidity and on broad-spectrum antibiotics. ${ }^{33,34}$ It is probable that the recent outbreak of CDAD was promoted by several causes, but particularly by association of PPIs with new and evolving patterns of broad-spectrum antimicrobial drugs, e.g., the fluoroquinolones. ${ }^{35,36}$

So CDAD should be considered in elderly hospitalized patients taking PPIs and broad-spectrum antibiotics developing refractory diarrhoea and, until trial data will be available, clinicians should consider the risks and benefits of continuing chronic PPIs therapy. Some experts recommend that all non-urgent PPIs therapy should be discontinued during hospitalization to minimize the risk of CDAD, while another proposal suggests continuing PPIs therapy when appropriately indicated at the lowest effective dose. ${ }^{37,38}$

In summary, multiple case-controlled and cohort studies have identified an association between PPIs exposure and the development of community-acquired and health care-associated CD infection (the risk of CDAD also exists in non-hospitalized patients on PPIs therapy but it has not been adequately studied). ${ }^{39-43}$ Nevertheless, further investigations are needed for a better definition of clinical consequences and therapeutic strategies. ${ }^{27}$

\section{Respiratory infections}

Pulmonary micro-aspiration of gastric contents, which may be infected as a consequence of the decreased gastric acid production, has led researchers to examine the relationship between AST and the development of community-acquired pneumonia (CAP). A case-controlled study performed on 5551 patients in the Netherlands observed a significant difference in CAP incidence among subjects currently taking PPIs compared with those who had stopped PPIs use [OR=1.89 (95\% CI 1.36-2.62)]..$^{44}$ Also H2RAs increased the relative risk of developing CAP, but less than PPIs $[\mathrm{OR}=1.63$ (95\% CI 1.07-2.48)]. Thus, in this study patients who were taking AST had a significant higher risk of developing CAP compared with those who had never taken AST. The risk of CAP development was found to be greatest among patients who began PPIs therapy within 30 days prior to CAP diagnosis.

The CAP increased risk among patients taking PPIs was confirmed in other studies. ${ }^{45,46}$ However, a CAP increased risk inversely proportional to the duration of PPIs use was also confirmed. The inverse relationship between the magnitude of CAP association and the duration of PPIs use in several studies, with 
the weakest association among patients who were prescribed a PPIs for the longest duration of time, is apparently surprising but it may be caused by a higher comorbidity in pneumonia cases on PPIs. When data were adjusted for confounding variables, no significant increase in pneumonia risk was demonstrated. ${ }^{46}$ A meta-analysis of seven randomized controlled trials evaluating efficacy of PPIs that recorded respiratory adverse events failed to show a significant association between PPIs and respiratory infections $(\mathrm{OR}=1.4$; 95\% CI, 0.9-2.3), even among ventilator-assisted patients on chronic PPIs therapy, in whom abnormal gastric colonization very often exists and the theoretical risk of increased microaspiration or translocation would be greatest. ${ }^{47}$

At last PPIs do not increase the risk of hospital acquired (nosocomial) pneumonia (NP). In fact, there is a reduced risk of NP in patients with nasogastric tubes on a PPIs. ${ }^{48}$ For ventilated pediatric patients in ICU, there is no increased risk of NP. ${ }^{49-51}$

Thus, we can conclude that long-term PPIs use do not increase the risk of CAP; short-term use could increase the risk of CAP but not of NP.

\section{Nutritional deficiencies}

\section{Vitamin $B_{12}$}

Gastric acidity is necessary to activate pepsinogen to pepsin leading to the release of vitamin B12 from B12-containing foods. The reduction of the proteinbound B12 absorption is usually very low when PPIs are used for a short-term. ${ }^{52-54}$ Prospective studies demonstrated decrease in vitamin $\mathrm{B}_{12}$ levels within the normal range, suggesting that the risk of deficiency may be clinically insignificant. ${ }^{55-57}$ Consumption of a normal diet will safeguard against clinically significant $B_{12}$ deficiency when taking a PPI. Many studies have shown that also PPIs used long term in non-elderly patients do not reduce seriously serum vitamin $\mathrm{B} 12$ concentrations and therefore body B12 stores. ${ }^{58-63}$ Thus, a significant reduction in serum vitamin B12 levels and a serious anaemia seem possible only in elderly patients with gastric atrophy ${ }^{54,64,65}$ and in patients with ZES, who need a long-term therapy at very high dosage. ${ }^{66}$

\section{Iron}

Patients with achlorhydria or significant gastric acid hyposecretion caused by vagotomy, gastric resection or atrophic gastritis may have iron deficiency anemia. Instead patients treated long-term with PPIs high doses have a small reduction in duodenal absorption of organic and non-organic iron and the risk of latent iron deficiency or iron deficiency is not increased. ${ }^{67,68}$ A cohort of patients with Zollinger-Ellison syndrome who were treated with PPIs for over 10 years did not develop significant iron deficiency. ${ }^{69}$

\section{Magnesium}

Hypomagnesemia is a rare event secondary to chronic PPIs therapy. Fewer than 30 cases have been described in the literature since 2006, with $61 \%$ having received PPIs therapy for 5 or more years and $29 \%$ for at least 10 years. ${ }^{55}$ However, it is important to keep in mind this possibility because hypomagnesemia could have severe clinical consequences (tiredness, instability, tetany, convulsions, cardiac arrhythmias and increased risk for hospitalization) and could be associated with hypopotassiemia and/or hypercalcemia. Patients with hypomagnesemia receiving both a PPI and a diuretic are at higher risk for hospitalization. ${ }^{70}$

Magnesium supplementation alone did not sufficiently increase serum magnesium levels and PPIs therapy had to be discontinued..$^{71}$ If AST is essential, switching to an H2RA should be considered a reasonable practice. ${ }^{26}$

There is no accepted biological mechanism to explain hypomagnesemia in PPIs users. Low-serum magnesium results from impaired absorption or intake, excessive losses (urinary or gastrointestinal), redistribution from extracellular to intracellular sites or acquired deficiencies in receptors of bivalent cations (calcium and magnesium) channels, subfamily $M$, member 6 (TRPM6) and member 7 (TRPM7). ${ }^{72}$ Increased gastric $\mathrm{pH}$ could affect the function of these channels.

\section{Rebound acid hypersecretion}

PPIs therapy increases G-cell release of circulating gastrin, which has trophic effect on oxyntic mucosa, causing hyperplasia and increased functional capacity of enterochromaffin-like (ECL) cells and parietal cells. ${ }^{55,73}$ So acid-related heartburn, acid regurgitation or dyspepsia could present again following drug cessation owing to increased acid secretion due to sustained hypergastrinemia. ${ }^{74,75}$ Some randomized double-blind trials have confirmed the possibility of symptomatic acid-related dyspepsia as a consequence of prolonged PPIs therapy and the increased risk of long-term hypergastrinemia, ${ }^{74,76}$ but at the moment still conflicting data exist on whether discontinuation of PPIs therapy is associated with rebound acid hypersecretion (RAH). It seems possible particularly in patients with diseases (e.g., GERD), which really need a long-term and uninterrupted therapy. For these conditions other therapeutic options previously indicated may be useful: use of the lowest effective dose for symptom control, try on-demand therapy, manage uncomplicated heartburn with antacids or H2RA, which cause negligible RAH and can be discontinued later with little difficulty. $75,77,78$

\section{Increased risk of gastric neoplasms}

During early animal safety tests on long-term omeprazole therapy, rats with hypergastrinemia devel- 
oped carcinoid tumors. However in humans hypergastrinemia alone may be associated with gastric ECL hyperplasia or redistribution, but it has not been associated with neoplastic changes. ${ }^{79}$ Only if coupled with other factors, such as genetic abnormality of multiple endocrine neoplasia type 1, long-standing hypergastrinemia can produce gastric ECL carcinoids in humans. ${ }^{79}$ Given the low incidence of gastric carcinoid tumors, prospective studies would not be feasible; thus, it is unlikely that the rat tumorigenic phenotypes will ever be reproduced in humans. ${ }^{26}$

PPIs use is associated with parietal cell hyperplasia and a fourfold increased incidence of fundic gland polyps (FGP) (Figure 2) ${ }^{80-82}$ FGPs occur in the presence or absence of $H$. pylori infection. ${ }^{83,84}$

Eradication of $H$. pylori or stopping long-term use of PPIs is associated with regression of FGPs. FGPs may become dysplastic rarely and only in patients with familial adenomatous polyposis. ${ }^{83,85,86}$ PPIs use has never caused gastric adenocarcinoma. ${ }^{87}$

\section{Osteopenia, osteoporosis and risk of bone fracture}

Is PPIs use associated with an increased risk of osteoporosis/osteopenia and, more importantly, with bone fracture? A certain answer to this question is a great challenge. ${ }^{88}$ No long-term prospective randomized, blinded, controlled trials exist addressing the potential increased risk of bone fracture concomitant with PPIs therapies, as the majority of existing data come from retrospective case-controlled, cohort and cross-sectional studies.

Several retrospective studies have demonstrated an increased risk in hip, spine, and wrist fractures in both men and women at highest risk for fractures among those who have taken increased PPIs doses for longer durations. ${ }^{27}$

In these case-control studies the association between prolonged PPIs use and increased bone fracture risk is weak, ranging from $20 \%$ to $60 \%$, with a low magnitude of ORs $(<2)$. Moreover, some confounding variables, that may bias results, are present. ${ }^{89-92}$ Of note, PPIs use is associated with greater than $50 \%$ increase in fracture risk among current and former smokers; in contrast, there is no association among women who never smoked. ${ }^{89}$

The results of other studies are different. PPIs use for up to 5 years is not associated with osteoporosis, even at a high dose, and marginally affects 3 -year bone mineral density of the hip in postmenopausal women. ${ }^{93,94}$ Kaye and Jick and Corley et al. examined PPIs use and bone fractures, adjusting for other key independent risk factors (i.e., alcohol abuse, arthritis, diabetes, kidney diseases, glucocorticoids, cerebrovascular diseases, dementia, epilepsy, visual impairment, anxiolytics and pre-existing osteoporosis), showing no increase in fractures. ${ }^{95,96}$ Corley et al. did demonstrate a $20 \%$ increase (OR 1.2; $95 \%$ CI, 1.2-1.3) in fracture risk among PPIs users when one or more other independent risk factors for bone fractures were present. ${ }^{96}$

However as many retrospective studies give contrasting results, the link between PPIs use and metabolic bone disease is really complex.

Indeed PPIs users have a strong acid suppression with triple effect of impairing vitamin $B_{12}$ and calcium absorption and causing hypergastrinemia. Impaired $\mathrm{B}_{12}$ absorption decreases osteoblastic activity and con-


Figure 2. Multiple fundic gland polyps in two patients during long-term therapy with proton pump inhibitors. Courtesy by Pietro Di Giorgio and Francesco Giannattasio, Unit of Digestive Endoscopy, Loreto Mare Hospital, Naples, Italy. 
sequently bone formation, while on the other hand increases homocysteine levels, which negatively affect collagen cross-linking, resulting in reduced metabolic bone density and bone strength. Hypergastrinemia causes parathyroid hormone release from hyperplastic parathyroid glands, contributing to increased bone absorption and decreased metabolic bone density and bone strength. Diminished calcium absorption negatively influences calcium homeostasis and reduces plasma calcium levels, turning on parathyroid hormone release with subsequent increase in circulating parathyroid hormone, furthering the decline in metabolic bone density and bone strength. ${ }^{26,73}$

Thus, it has been recommended that older patients who require long-term or high-dose PPIs therapy should consider increased dietary or supplementary calcium and vitamin D intake to minimize risks of bone fracture. ${ }^{27}$ This advice could be useful also for postmenopausal women.

\section{Interstitial nephritis}

PPIs are listed among the drugs, which are cause of about $60 \%$ of acute interstitial nephritis (AIN), an immunomediate disease with inflammation of the renal interstitium and tubules, and possible progress to acute renal failure. ${ }^{72,97}$ PPIs-related AIN is rare, idiosyncratic and, therefore, difficult to predict. Its exact incidence is not known because literature data are limited to observational cases or small series (altogether 60 cases). ${ }^{97} \mathrm{All}$ PPIs have been associated with AIN, but the most commonly implicated is omeprazole, and the least is rabeprazole ${ }^{97}$ Obviously diagnosis and supposition of AIN oblige to the suspension of PPIs therapy.

\section{Interaction between proton pump inhibitors and other drugs}

Some risks, in addition to iatrogenic diseases above mentioned, are related to the PPIs interaction with other drugs. ${ }^{88}$ Achlorydria or severe hypochlorydria reduce the effects of drugs, as ketoconazole, itraconazole and indinipur, requiring intragastric acidity to maximize their absorption and bioavailability. ${ }^{98}$ PPIs also influence drug absorption and metabolism by interacting with adenosine triphosphatedependent P-glycoprotein (e.g., inhibiting digoxin efflux) or with the cytochrome P450 (CYP) enzyme system (e.g., decreasing simvastatin metabolism), thereby affecting both intestinal first-pass metabolism and hepatic clearance. This effect is greater for omeprazole, because its high affinity for CYP2C19 isoenzyme and a somewhat lower affinity for CYP3A4 isoenzyme, than for pantoprazole, which appears to have lower interactions with other medications. The interaction profiles of esomeprazole, lansoprazole and rabeprazole have been less investigated, but evidence suggests that lansoprazole and rabeprazole seem to have a weaker potential for interactions than omeprazole.

A recent study has documented that in 5-6\% of patients on treatment with both PPI and L-thyroxine, the interaction between the two drugs is significant, causing an increase in serum thyroid stimulating hormone $>5 \mathrm{mU} / \mathrm{L}$ and, thus, the relapse of a clinical and biochemical pattern of hypothyroidism. ${ }^{99}$ The rigorous methodology of this study probably allows overcoming the existing uncertainties about the interaction between L-thyroxine and PPI. However, it does not clarify the mechanism of such interaction, which may not necessarily be the increase of L-thyroxine absorption caused by high gastric $\mathrm{pH}$.

For the time being the biggest problem is the interaction between PPIs and antiplatelets agents. Clopidogrel in association with aspirin is considered state of the art medical treatment for acute coronary syndrome by reducing the risk of new ischemic events. Concomitant treatment with PPIs in order to prevent gastrointestinal side effects is recommended. Clopidogrel needs metabolic activation predominantly by the hepatic CYP2C19 isoenzyme, which extensively metabolize PPIs, as mentioned above.

Several trials have evaluated the reduced effectiveness of clopidogrel in patients taking concomitant PPIs therapy. ${ }^{100-102}$ These studies have not permitted to provide an evidence-based recommendation about PPIs therapy in patients receiving antiplatelet therapy either during an acute coronary syndrome (ACS) or during maintenance therapy after an ACS or a cerebrovascular accident. Additional research is needed to further examine this issue in larger cohorts, with headto-head comparisons across all PPIs, as well as with newer antiplatelets agents.

However these observations led the United States Food and Drug Administration (FDA) and the European Medicines Agency (EMA) to discourage the combination of clopidogrel and PPIs (especially omeprazole). ${ }^{103,104}$ The FDA also warned that separating the administration times of clopidogrel and omeprazole did not reduce drug interaction. In patients with high risk of gastrointestinal bleeding, combination of clopidogrel with the less CYP2C19 inhibiting pantoprazole should be recommended. ${ }^{105}$ To date, cimetidine is the only H2RA known to interact with clopidogrel. $^{27}$

Recently a retrospective study, ${ }^{106}$ analyzing 2.9 million patient data, has been published suggesting that PPIs therapies are associated with an increased risk of myocardial infarction in the general population.

\section{Conclusions}

PPIs have revolutionized the therapy of numerous upper GI tract disorders, but they account for a signif- 
icant proportion of pharmaceutical health-care expenditure, most unjustified because PPIs are inappropriately prescribed to a great number of in- and outpatients. In addition to the wrong prescriptions, the excessive expenditure is often caused by prolonging the therapy further the real necessities. As for all medications, the risks of using long-term PPIs must be compared with the benefits, weighing accurately more factors related to the disease and the patient. Also for the diseases (e.g., GERD) justifying a long-term therapy, it is possible to search for alternative treatments (step-down or on-demand PPIs administration, therapeutic intervals with other drugs).

However, PPIs therapy is not without risk of adverse effects. The possibility and the incidence of some iatrogenic diseases related to PPIs use are not well defined yet. Large randomized, prospective trials are needed to establish more accurately cause/effect relationships of adverse events during PPIs therapy.

\section{References}

1. Mendoza Ladd AM, Panagopoulos G, Cohen J, et al. Potential costs of inappropriate use of proton pump inhibitors. Am J Med Sci 2014;347:446-51.

2. Ahrens D, Chenot JF, Behrens G, et al. Appropriateness of treatment recommendations for PPI in hospital discharge letters. Eur J Clin Pharmacol 2010;66:1265-71.

3. Brooks M. Top 100 most prescribed, top selling drugs. MEDSCAPE Intern Med; May 13, 2014. Available from: http://www.medscape.com/viewarticle/825053

4. National Institute for Clinical Excellence (NICE). Guidance on the use of proton pump inhibitors (PPI) in the treatment of dyspepsia. NICE technology appraisal guidance [TA7]; 2000. Available from: https://www. nice.org.uk/guidance/ta7

5. Haroon M, Yasin F, Gardezi SK, et al. Inappropriate use of proton pump inhibitors among medical inpatients: a questionnaire-based observational study. JRSM Short Rep 2013;4:2042533313497183.

6. Sebastian SS, Kernan N, Qasim A, et al. Appropriateness of gastric antisecretory therapy in hospital practice. Ir J Med Sci 2003;172:115-7.

7. Pham CQD, Regal RE, Bostwick TR, Knauf KS. Acid suppressive therapy use on an inpatient internal medicine service. Ann Pharmacother 2006;40:1261-6.

8. Bjornsson E, Abrahamsson H, Simren M, et al. Discontinuation of proton pump inhibitors in patients on long term therapy: a double blind, placebo controlled trial. Aliment Pharmacol Ther 2006;24:945-54.

9. Batuwitage B, Kingham JCG, Morgan NE, Bartlett RL Inappropriate prescribing of proton pump inhibitors in primary care. Postgrad Med J 2007;83:66-8.

10. American Society of Health-System Pharmacists. ASHP therapeutic guidelines on stress ulcer prophylaxis. Am J Health-Syst Pharm 1999;56:347-79.

11. Heidelbaugh JJ, Goldberg KL, Pharm D, Inadomi JM. Overutilization of proton pump inhibitors: a review of cost-effectiveness and risk in PPI. Am J Gastroenterol 2009;104:S27-32.
12. Cash BD. Evidence-based medicine as it applies to acid suppression in the hospitalized patient. Crit Care Med 2002;30:S373-8.

13. Heidelbaugh JJ, Inadomi JM. Magnitude and economic impact of inappropriate use of stress ulcer prophylaxis in non-intensive care unit hospitalized patients. Am J Gastroenterol 2006;101:2200-5.

14. Nardino RJ, Vender RJ, Herbert PN. Overuse of acidsuppressive therapy in hospitalized patients. Am J Gastroenterol 2000;95:3118-22.

15. Zink DA, Pohlman M, Barnes M, et al. Long-term use of acid suppression started inappropriately during hospitalization. Aliment Pharmacol Ther 2005;21:1203-9.

16. Hussain S, Stefan M, Visintainer, Rothberg M. Why do physicians prescribe stress ulcer prophylaxis to general medicine patients? South Med J 2010;103:1103-10.

17. Heidelbaugh JJ, Goldberg KL, Inadomi JM. Magnitude and economic impact of overutilization of antisecretory therapy in the ambulatory care setting. Am J Manag Care 2010;16:228-34.

18. Jacobson BC, Ferris TG, Shea TL, et al. Who is using chronic acid suppression therapy and why? Am J Gastroenterol 2003;98;51-8.

19. Inadomi JM, McIntyre L, Bernard L, et al. Step-down from multiple to single-dose proton pump inhibitors (PPIs): a prospective study of patients with heartburn or acid regurgitation completely relieved with PPIs. Am J Gastroenterol 2003;98:1940.

20. Lind T, Havelund T, Lundell L, et al. On demand therapy with omeprazole for the long-term management of patients with heartburn without oesophagitis - a placebo-controlled randomized trial. Aliment Pharmacol Ther 1999;13:907-14.

21. Talley NJ, Lauritsen K, Tunturi-Hihnala H, et al. Esomeprazole $20 \mathrm{mg}$ maintains symptom control in endoscopy-negative gastro-oesophageal reflux disease: a controlled trial of 'on-demand' therapy for 6 months. Aliment Pharmacol Ther 2001;15:347-54.

22. Talley NJ, Venables TL, Green JR, et al. Esomeprazole $40 \mathrm{mg}$ and $20 \mathrm{mg}$ is efficacious in the long-term management of patients with endoscopy-negative gastrooesophageal reflux disease: a placebo-controlled trial of on-demand therapy for 6 months. Eur J Gastroenterol Hepatol 2002;14:857-63.

23. Bytzer P, Blum A, De Herdt D, et al. Six-month trial of on-demand rabeprazole $10 \mathrm{mg}$ maintains symptom relief in patients with non-erosive reflux disease. Aliment Pharmacol Ther 2004;20:181-8.

24. Inadomi JM, Jamal R, Murata GH, et al. Step-down management of gastroesophageal reflux disease. Gastroenterology 2001;121:1095-100.

25. Johnsson F, Moum B, Vilien M, et al. On-demand treatment in patients with oesophagitis and reflux symptoms: comparison of lansoprazole and omeprazole. Scand J Gastroenterol 2002;37:642-7.

26. National Institute for Clinical Excellence (NICE). Dyspepsia and gastro-oesophageal reflux disease: investigation and management of dyspepsia, symptoms suggestive of gastro- oesophageal reflux disease, or both. NICE guidelines [CG184]; September 2014. Available from: https://www.nice.org.uk/guidance/cg184

27. Abraham NS. Proton pump inhibitors: potential adverse effects. Curr Opin Gastroenterol 2012;28:615-20. 
28. Heidelbaugh JJ, Kim AH, Chang R, Walker PC. Overutilization of proton-pump inhibitors: what the clinician needs to know. Therap Adv Gastroenterol 2012; 5:219-32.

29. Howden CW, Hunt RH. Relationship between gastric secretion and infection. Gut 1987;28:96-107.

30. Leonard J, Marshall JK, Moayyedi P. Systematic review of the risk of enteric infection in patients taking acid suppression. Am J Gastroenterol 2007;102:2047-56.

31. Elphick DA, Chew TS, Higham SE, et al. Small bowel bacterial overgrowth in symptomatic older people: can it be diagnosed earlier? Gerontology 2005;51:396-401.

32. Williams C, McColl KE. Review article: proton pump inhibitors and bacterial overgrowth. Aliment Pharmacol Ther 2006;23:3-10.

33. Deshpande A, Pant C, Pasupuleti V, et al. Association between proton pump inhibitor therapy and Clostridium difficile infection in a meta-analysis. Clin Gastroenterol Hepatol 2012;10:225-33.

34. Linsky A, Gupta K, Lawler EV, et al. Proton pump inhibitors and risk for recurrent Clostridium difficile infection. Arch Intern Med 2010;170:772-8.

35. Yip C, Loeb M, Salama S, et al. Quinolone use as a risk factor for nosocomial Clostridium difficile-associated diarrhea. Infect Control Hosp Epidemiol 2001;22:572-5.

36. McCusker ME, Harris AD, Perencevich E, Roghmann MC. Fluoroquinolone use and Clostridium difficile-associated diarrhea. Emerg Infect Dis 2003;9:730-3.

37. Thachil J. Overprescribing PPIs: time for a hospital antacid policy on Clostridium difficile. BMJ 2008; 336:109.

38. Metz DC. Clostridium difficile colitis: wash your hands before stopping the proton pump inhibitor. Am J Gastroenterol 2008;103:2314-16.

39. Cunningham R, Dale B, Undy B, Gaunt N. Proton pump inhibitors as a risk factor for Clostridium difficile diarrhoea. J Hosp Infect 2003;54:243-5.

40. Dial S, Alrasadi K, Manoukian C, et al. Risk of Clostridium difficile diarrhea among hospital inpatients prescribed proton pump inhibitors: cohort and casecontrol studies. CMAJ 2004;171:33-8.

41. Jayatilaka S, Shakov R, Eddi R, et al. Clostridium difficile infection in an urban medical center: five-year analysis of infection rates among adult admissions and association with the use of proton pump inhibitors. Ann Clin Lab Sci 2007;37:241-7.

42. Aseeri M, Schroeder T, Kramer J, Zackula R. Gastric acid suppression by proton pump inhibitors as a risk factor for Clostridium-difficile-associated diarrhea in hospitalized patients. Am J Gastroenterol 2008;103: 2308-13.

43. Loo VG, Bourgault AM, Poirier L, et al. Host and pathogen factors for Clostridium difficile infection and colonization. N Eng J Med 2011;365:1693-703.

44. Laheij RJF, Sturkenboom MCJM, Hassing RJ, et al. Risk of community-acquired pneumonia and use of gastric acid-suppressive drugs. JAMA 2004;292:1955-60.

45. Gulmez SE, Holm A, Frederiksen H, et al. Use of proton pump inhibitors and the risk of community-acquired pneumonia: a population-based case-control study. Arch Int Med 2007;167:950-5.

46. Sarkar M, Hennessy S, Yang YX. Proton-pump inhibitor use and the risk for community-acquired pneumonia. Ann Int Med 2008;149:391-8.
47. Sultan N, Nazareno J, Gregor J. Association between proton pump inhibitors and respiratory infections: a systematic review and meta-analysis of clinical trials. Can J Gastroenterol 2008;22:761-6.

48. Yamanaka Y, Mammoto T, Kita T, Kishi Y. A study of 13 patients with gastric tube in place after esophageal resection: use of omeprazole to decrease gastric acidity and volume. J Clin Anesth 2001;13:370-3.

49. Yildizdas D, Yapicioglu H, Yilmaz HL. Occurrence of ventilator-associated pneumonia in mechanically ventilated pediatric intensive care patients during stress ulcer prophylaxis with sucralfate, ranitidine, and omeprazole. J Crit Care 2002;17:240-5.

50. Levy MJ, Seelig CB, Robinson NJ, Ranney JE. Comparison of omeprazole and ranitidine for stress ulcer prophylaxis. Dig Dis Sci 1997;42:1255-9.

51. Kantorova I, Svoboda P, Scheer P, et al. Stress ulcer prophylaxis in critically ill patients: a randomized controlled trial. Hepatogastroenterology 2004;51:757-61.

52. Marcuard SP, Albernaz L, Khazanie PG. Omeprazole therapy causes malabsorption of cyanocobalamin (vitamin B12) Ann Intern Med 1994;120:211-5.

53. Saltzman JR, Kemp JA, Golner BB, et al. Effect of hypochlorhydria due to omeprazole treatment or atrophic gastritis on protein-bound vitamin B12 absorption. J Am Coll Nutr 1994;13:584-91.

54. Schenk BE, Festen HP, Kuipers EJ, et al. Effect of short- and long-term treatment with omeprazole on the absorption and serum levels of cobalamin. Aliment Pharmacol Ther 1996;10:541-5.

55. Sheen E, Triadafilopoulos G. Adverse effects of longterm proton pump inhibitor therapy. Dig Dis Sci 2011;56:931-50.

56. Dharmarajan TS, Kanagala MR, Murakonda P, et al. Do acid-lowering agents affect vitamin B12 status in older adults? J Am Med Dir Assoc 2008;9:162-7.

57. Rozgony NR, Fang C, Kuczmarski MF, Bob H. Vitamin $\mathrm{B}(12)$ deficiency is linked with long-term use of proton pump inhibitors in institutionalized older adults: could a cyanocobalamin nasal spray be beneficial? J Nutr Elder 2010;29:87-99.

58. Kapadia C. Cobalamin (Vitamin B12) deficiency: is it a problem for our aging population and is the problem compounded by drugs that inhibit gastric acid secretion? J Clin Gastroenterol 2000;30:4-6.

59. Wolters M, Ströhle A, Hahn A. Cobalamin: a critical vitamin in the elderly. Prev Med 2004;39:1256-66.

60. den Elzen WP, Groeneveld Y, de Ruijter W, et al. Longterm use of proton pump inhibitors and vitamin B12 status in elderly individuals. Aliment Pharmacol Ther 2008;27:491-7.

61. Howden CW. Vitamin B12 levels during prolonged treatment with proton pump inhibitors. J Clin Gastroenterol 2000;30:29-33.

62. Maton PN, Vinayek R, Frucht H, et al. Long-term efficacy and safety of omeprazole in patients with Zollinger-Ellison syndrome: a prospective study. Gastroenterology 1989;97:827-36.

63. Mitchell SL, Rockwood K. The association between antiulcer medication and initiation of cobalamin replacement in older persons. J Clin Epidemiol 2001;54: 531-4.

64. Ruscin JM, Page RL, Valuck RJ. Vitamin B(12) deficiency associated with histamine(2)-receptor antago- 
nists and a proton-pump inhibitor. Ann Pharmacother 2002; 36:812-6.

65. Schenk BE, Kuipers EJ, Klinkenberg-Knol EC, et al. Atrophic gastritis during long-term omeprazole therapy affects serum vitamin B12 levels. Aliment Pharmacol Ther 1999;13:1343-6.

66. Termanini B, Gibril F, Sutliff VE, et al. Effect of longterm gastric acid suppressive therapy on serum vitamin B12 levels in patients with Zollinger-Ellison syndrome. Am J Med 1998;104:422-30.

67. Sharma VR, Brannon MA, Carloss EA. Effect of omeprazole on oral iron replacement in patients with iron deficiency anemia. South Med J 2004;97:887-9.

68. Koop H, Bachem MG. Serum iron, ferritin, and vitamin B12 during prolonged omeprazole therapy. J Clin Gastroenterol 1992;14:288-92.

69. Stewart CA, Termanini B, Sutliff VE, et al. Iron absorption in patients with Zollinger-Ellison syndrome treated with long-term gastric acid antisecretory therapy. Aliment Pharmacol Ther 1998;12:83-98.

70. Henderson D. PPI and diuretic up risk with hypomagnesemia hospitalization. PLoS Med 2014;11:e1001736.

71. Food and Drug Administration (FDA). FDA Drug Safety Communication: Low magnesium levels can be associated with long-term use of proton pump inhibitor drugs (PPIs); December 16, 2011. Available from: http://www.fda.gov/drugs/drugsafety/ucm245011.htm

72. Yang YX, Metz DC. Safety of proton pump inhibitor exposure. Gastroenterology 2010;139:1115-27.

73. McColl KE, Gillen D. Evidence that proton-pump inhibitor therapy induces the symptoms it is used to treat. Gastroenterology 2009;137:20-2.

74. Reimer C, Sondergaard B, Hilsted L, Bytzer P. Protonpump inhibitor therapy induces acid-related symptoms in healthy volunteers after withdrawal of therapy. Gastroenterology 2009;137:80-77.

75. McCarthy DM. Adverse effects of proton pump inhibitor drugs: clues and conclusions. Curr Opin Gastroenterol 2010;26:624-31.

76. Niklasson A, Lindstrom L, Simren M, et al. Dyspeptic symptom development after discontinuation of a proton pump inhibitor: a double-blind placebocontrolled trial. Am J Gastroenterol 2010;105:1531-7.

77. Metz DC, Pilmer BL, Han C, Perez MC. Withdrawing PPI therapy after healing esophagitis does not worsen symptoms or cause persistent hypergastrinemia: analysis of dexlansoprazole MR clinical trial data. Am J Gastroenterol 2011;106:1953-60.

78. Kahrilas PJ, Shaheen NJ, Vaezi MF, et al. American Gastroenterological Association medical position statement on the management of gastroesophageal reflux disease. Gastroenterology 2008;135:1383-91.

79. Laine L, Ahnen D, McClain C, et al. Review article: potential gastrointestinal effects of long-term acid suppression with proton pump inhibitors. Aliment Pharmacol Ther 2000;14:651-68.

80. el-Zimaity HM, Jackson FW, Graham DY. Fundic gland polyps developing during omeprazole therapy. Am J Gastroenterol 1997;92:1858-60.

81. Raghunath AS, O'Morain C, McLoughlin RC. Review article: the long-term use of proton-pump inhibitors. Aliment Pharmacol Ther 2005;22:55-63.

82. Jalving M, Koornstra JJ, Wesseling J, et al. Increased risk of fundic gland polyps during long-term proton pump inhibitor therapy. Aliment Pharmacol Ther 2006;24:1341-8.

83. Wu TT, Kornacki S, Rashid A, et al. Dysplasia and dysregulation of proliferation in foveolar and surface epithelia of fundic gland polyps from patients with familial adenomatous polyposis. Am J Surg Pathol 1998;22:293-8.

84. Sakai N, Tatsuta M, Hirasawa R, et al. Low prevalence of Helicobacter pylori infection in patients with hamartomatous fundic polyps. Dig Dis Sci 1998;43:766-72.

85. Watanabe N, Seno H, Nakajima T, et al. Regression of fundic gland polyps following acquisition of Helicobacter pylori. Gut 2002;51:742-5.

86. Bertoni G, Sassatelli R, Nigrisoli E, et al. Dysplastic changes in gastric fundic gland polyps of patients with familial adenomatous polyposis. Ital J Gastroenterol Hepatol 1999;31:192-7.

87. Jalving M, Koornstra JJ, Götz JM, et al. High-grade dysplasia in sporadic fundic gland polyps: a case report and review of the literature. Eur J Gastroenterol Hepatol 2003; 15:1229-33.

88. Thomson ABR, Sauve MD, Kassam N, Kamitakahara H. Safety of the long-term use of proton pump inhibitors. World J Gastroenterol 2010;16:2323-30.

89. Khalili H, Huang ES, Jacobson BC, et al. Use of proton pump inhibitors and risk of hip fracture in relation to dietary and lifestyle factors: a prospective cohort study. BMJ 2012;344:e372.

90. Targownik LE, Lix LM, Metge CJ, et al. Use of proton pump inhibitors and risk of osteoporosis-related fractures. CMAJ 2008;179:319-26.

91. Vestergaard P, Rejnmark L, Mosekilde L. Proton pump inhibitors, histamine $\mathrm{H} 2$ receptor antagonists, and other antacid medications and the risk of fracture. Calcif Tissue Int 2006;79:76-83.

92. Yang YX, Lewis JD, Epstein S, Metz DC. Long-term proton pump inhibitor therapy and risk of hip fracture. JAMA 2006;296:2947-53.

93. Targownik LE, Lix LM, Leung S, Leslie WD. Protonpump inhibitor use is not associated with osteoporosis or accelerated bone mineral density loss. Gastroenterology 2010;138:896-904.

94. Gray SL, LaCroix AZ, Larson J, et al. Proton pump inhibitor use, hip fracture, and change in bone mineral density in postmenopausal women: results from the Women's Health Initiative. Arch Intern Med 2010;170: 765-71.

95. Kaye JA, Jick H. Proton pump inhibitor use and risk of hip fractures in patients without major risk factors. Pharmacotherapy 2008;28:951-9.

96. Corley DA, Kubo A, Zhao W, Quesenberry C. Proton pump inhibitors and histamine-2-receptor antagonists are associated with hip fractures among at risk patients. Gastroenterology 2010;139:93-101.

97. Sierra F, Suarez M, Rey M, Vela MF. Systematic review: proton pump inhibitor associated acute interstitial nephritis. Aliment Pharmacol Ther 2007;26:545-53.

98. Blume H, Donath F, Warnke A, Schug BS. Pharmacokinetic drug interaction profiles of proton pump inhibitors. Drug Saf 2006;29:769-84.

99. Irving SA, Vadiveloo T, Leese GP. Drugs that interact with levothyroxine an observational study from the thy- 
roid epidemiology, audit and research study (TEARS). Clin Endocrinol 2015;82:136-41.

100. Gilard M, Arnaud B, Cornily JC, et al. Influence of omeprazole on the antiplatelet action of clopidogrel associated with aspirin. The randomized, double-blind OCLA (omeprazole clopidogrel aspirin) study. J Am Coll Cardiol 2008;51:256-60.

101. Siller-Matula JM, Spiel AO, Lang IM, et al. Effects of pantoprazole and esomeprazole on platelet inhibition by clopidogrel. Am Heart J 2009;157:148.e1-5.

102. Cuisset T, Frere C, Quilici J, et al. Comparison of omeprazole and pantoprazole influence on a high 150mg clopidogrel maintenance dose. The PACA (proton pump inhibitors and clopidogrel association) prospective randomized study. J Am Coll Cardiol 2009;54: 1149-53.

103. Food and Drug Administration (FDA). Public Health Ad- visory: Updated safety information about a drug interaction between clopidogrel bisulfate (marketed as Plavix) and omeprazole (marketed as Prilosec and Prilosec OTC); November 17, 2009. Available from: http://www. fda.gov/Drugs/DrugSafety/PostmarketDrugSafetyInformationforPatientsandProviders/ucm 190825.htm

104. European Medicines Agency (EMA). Public statement on possible interaction between clopidogrel and proton pump inhibitors. EMEA/328956/2009. London: EMA; May 29, 2009.

105. Drepper MD, Spahr L, Frossard JL. Clopidogrel and proton pump inhibitors-where do we stand in 2012 ? World J Gastroenterol 2012;18:2161-71.

106. Shah NH, LePendu P, Bauer-Mehren A, et al. Proton pump inhibitor usage and the risk of myocardial infarction in the general population. PLoS ONE 2015 [Epub ahead of print]. 